\title{
Geodesic Flow and Two (Super) Component Analog of the Camassa-Holm Equation
}

\author{
Partha GUHA ${ }^{\dagger}$ and Peter J. OLVER ${ }^{\ddagger}$ \\ $\dagger$ S.N. Bose National Centre for Basic Sciences, JD Block, Sector-3, Salt Lake, \\ Calcutta-700098, India \\ E-mail: partha@bose.res.in \\ $\ddagger$ School of Mathematics, University of Minnesota, Minneapolis, MN 55455, USA \\ E-mail: olver@math.umn.edu \\ URL: http://www. math. umn.edu/ olver/
}

Received March 08, 2006, in final form May 08, 2006; Published online May 23, 2006

Original article is available at http://www.emis.de/journals/SIGMA/2006/Paper054/

\begin{abstract}
We derive the 2-component Camassa-Holm equation and corresponding $N=1$ super generalization as geodesic flows with respect to the $H^{1}$ metric on the extended BottVirasoro and superconformal groups, respectively.
\end{abstract}

Key words: geodesic flow; diffeomorphism; Virasoro orbit; Sobolev norm

2000 Mathematics Subject Classification: 53A07; 53B50

\section{Introduction}

About ten years ago, Rosenau, [26], introduced a class of solitary waves with compact support as solutions of certain wave equations with nonlinear dispersion. It was found that the solutions of such systems unchanged from collision and were thus called compactons. The discovery that solitons may compactify under nonlinear dispersion inspired further investigation of the role of nonlinear dispersion. It has been known for some time that nonlinear dispersion causes wave breaking or lead to the formation of corners or cusps. Beyond compactons, a wide variety of other exotic non-analytic solutions, including peakons, cuspon, mesaons, etc., have been found in to exist in a variety of models that incorporate nonlinear dispersion [15].

We will study integrable evolution equations appearing in bi-Hamiltonian form

$$
u_{t}=J_{1} \frac{\delta H_{1}}{\delta u}=J_{2} \frac{\delta H_{0}}{\delta u}, \quad n=0,1,2, \ldots,
$$

where $J_{1}$ and $J_{2}$ are compatible Hamiltonian operators. The initial Hamiltonians $H_{0}, H_{1}$ are the first two in a hierarchy of conservation laws whose corresponding bi-Hamiltonian flows are successively generated by the recursion operator

$$
\mathcal{R}=J_{2} J_{1}^{-1} .
$$

We refer the reader to [21] for the basic facts about bi-Hamiltonian systems.

In an earlier work, the second author showed with Rosenau [22] that a simple scaling argument shows that most integrable bi-Hamiltonian systems are governed by tri-Hamiltonian structures. They formulated a method of "tri-Hamiltonian duality", in which a recombination of the Hamiltonian operators leads to integrable hierarchies endowed with nonlinear dispersion that supports compactons or peakons. A related construction can be found in the contemporaneous paper of Fuchssteiner [8]. 
The tri-Hamiltonian formalism can be best described through examples. The Kortewegde Vries equation

$$
u_{t}=u_{x x x}+3 u u_{x}
$$

can be written in bi-Hamiltonian form (1) using the two compatible Hamiltonian operators

$$
J_{1}=D, \quad J_{2}=D^{3}+u D+D u, \quad \text { where } \quad D \equiv \frac{d}{d x}
$$

and

$$
H_{1}=\frac{1}{2} \int u^{2} d x, \quad H_{2}=\frac{1}{2} \int\left(-u_{x}^{2}+u^{3}\right) d x .
$$

The tri-Hamiltonian duality construction is implemented as follows:

- A simple scaling argument shows that $J_{2}$ is in fact the sum of two compatible Hamiltonian operators, namely $K_{2}=D^{3}$ and $K_{3}=u D+D u$, so that $K_{1}=J_{1}, K_{2}, K_{3}$ form a triple of mutually compatible Hamiltonian operators.

- Thus, when we can recombine the Hamiltonian triple by transferring the leading term $D^{3}$ from $J_{2}$ to $J_{1}$, thereby constructing the Hamiltonian pairs $\widehat{J}_{1}=K_{2} \pm K_{1}=D^{3} \pm D$. The resulting self-adjoint operator $S=1 \pm D^{2}$ is used to define the new field variable $\rho=S u=u \pm u_{x x}$.

- Finally, the second Hamiltonian structure is constructed by replacing $u$ by $\rho$ in the remaining part of the original Hamiltonian operator $K_{3}$, so that $\widehat{J}_{2}=\rho D+D \rho$. Note that this change of variables does not affect $\widehat{J_{1}}$.

As a result of this procedure, we recover the tri-Hamiltonian dual of the KdV equation

$$
\rho_{t}=\widehat{J}_{1} \frac{\delta \widehat{H}_{2}}{\delta \rho}=\widehat{J}_{2} \frac{\delta \widehat{H}_{1}}{\delta \rho}
$$

where

$$
\widehat{H}_{1}=\frac{1}{2} \int u \rho d x=\frac{1}{2} \int\left(u^{2} \mp u_{x}^{2}\right) d x, \quad \widehat{H}_{2}=\frac{1}{2} \int\left(u^{3} \mp u u_{x}^{2}\right) d x .
$$

In this case, (2) reduces to the celebrated Camassa-Holm equation [2, 3]:

$$
u_{t} \pm u_{x x t}=3 u u_{x} \pm\left(u u_{x x}+\frac{1}{2} u_{x}^{2}\right)_{x} .
$$

Remark 1. The choice of plus sign leads to an integrable equation which supports compactons, whereas the minus sign is the water wave model derived by Camassa-Holm, whose solitary wave solutions have a sharp corner at the crest.

The Ito equation. Let us next study the Ito equation [14],

$$
\begin{aligned}
& u_{t}=u_{x x x}+3 u u_{x}+v v_{x} \\
& v_{t}=(u v)_{x}
\end{aligned}
$$

which is a prototypical example of a two-component $\mathrm{KdV}$ equation. This can also be expressed in bi-Hamiltonian form using the following two Hamiltonian operators

$$
J_{1}=\left(\begin{array}{cc}
D & 0 \\
0 & D
\end{array}\right), \quad J_{2}=\left(\begin{array}{cc}
D^{3}+u D+D u & v D \\
D v & 0
\end{array}\right),
$$


with Hamiltonians

$$
H_{1}=\frac{1}{2} \int\left(u^{2}+v^{2}\right) d x, \quad H_{2}=\frac{1}{2} \int\left(u^{3}+u v^{2}-u_{x}^{2}\right) d x .
$$

Again, a simple scaling argument is used to split

$$
J_{2}=\left(\begin{array}{cc}
D^{3} & 0 \\
0 & 0
\end{array}\right)+\left(\begin{array}{cc}
u D+D u & v D \\
D v & 0
\end{array}\right)
$$

as a sum of two compatible Hamiltonian operators. To construct the dual, we transfer the leading term $D^{3}$ from the first Hamiltonian operator to the second. We obtain the first Hamiltonian operator for the new equation

$$
\widehat{J}_{1}=\left(\begin{array}{cc}
D \pm D^{3} & 0 \\
0 & D
\end{array}\right) \equiv D\left(\begin{array}{cc}
S & 0 \\
0 & 1
\end{array}\right) \text {. }
$$

Therefore, the new variables are defined as

$$
\left(\begin{array}{l}
\rho \\
\sigma
\end{array}\right)=\left(\begin{array}{ll}
S & 0 \\
0 & 1
\end{array}\right)\left(\begin{array}{l}
u \\
v
\end{array}\right)
$$

The second Hamiltonian structure follows from the truncated part of the original Hamiltonian operator $J_{2}$, so that

$$
\widehat{J}_{2}=\left(\begin{array}{cc}
\rho D+D \rho & v D \\
D v & 0
\end{array}\right)
$$

with

$$
\widehat{H}_{1}=\frac{1}{2} \int\left(u \rho+v^{2}\right) d x, \quad \widehat{H}_{2}=\frac{1}{2} \int\left(u^{3}+u v^{2} \mp u u_{x}^{2}\right) d x .
$$

The dual system (2) takes the explicit form

$$
\begin{aligned}
& u_{t} \pm u_{x x t}=3 u u_{x}+v v_{x}+\left(u u_{x x}+\frac{1}{2} u_{x}^{2}\right)_{x}, \\
& v_{t}=(u v)_{x} .
\end{aligned}
$$

Motivation. The Camassa-Holm equation was derived physically as a shallow water wave equation by Camassa and Holm [2, 3, 13], and identified as the geodesic flow on the group of onedimensional volume-preserving diffeomorphisms under the $H^{1}$ metric. The multi-dimensional analogs lead to important alternative models to the classical Euler equations of fluid mechanics. Later, Misiolek [20] showed that, like the KdV equation [23], it can also be characterized as a geodesic flow on the Bott-Virasoro group.

Recently, a 2-component generalization of the Camassa-Holm equation has drawn a lot of interest among researchers. Chen, Dubrovin, Falqui, Grava, Liu and Zhang (the group at SISSA) have been working on multi-component analogues, using reciprocal transformations and studying their effect on the Hamiltonian structures, $[4,7,16]$. They show that the 2-component system cited above admits peakons, albeit of a different shape owing to the difference in the corresponding Green's functions. Another two-component generalization also appeared recently as the bosonic sector of the extended $N=2$ supersymmetric Camassa-Holm equation [25].

Following Ebin-Marsden [6], we enlarge Diff $\left(S^{1}\right)$ to a Hilbert manifold Diff ${ }^{s}\left(S^{1}\right)$, the diffeomorphisms of the Sobolev class $H^{s}$. This is a topological space. If $s>n / 2$, it makes sense to talk about an $H^{s}$ map from one manifold to another. Using local charts, one can check whether 
the derivations of order $\leq s$ are square integrable. The Lie algebra of $\operatorname{Diff}^{s}\left(S^{1}\right)$ is denoted by $\operatorname{Vect}^{s}\left(S^{1}\right)$.

In this paper we show that a 2-component generalization of the Camassa-Holm equation and its super analog also follow from the geodesic with respect to the $H^{1}$ metric on the semidirect product space $\operatorname{Diff}^{s}\left(S^{1}\right) \ltimes C^{\infty}\left(S^{1}\right)$ and its supergroup respectively. In fact, it is known that numerous coupled KdV equations [10, 11, 12] follow from geodesic flows of the right invariant $L^{2}$ metric on the semidirect product group $\operatorname{Diff}\left(S^{1) \ltimes C} \infty\left(S^{1}\right)[1,18]\right.$.

\section{Preliminaries}

The Lie algebra of $\operatorname{Diff}^{s}\left(S^{1}\right) \ltimes C^{\infty}\left(S^{1}\right)$ is the semidirect product Lie algebra

$$
\mathfrak{g}=\operatorname{Vect}^{s}\left(S^{1}\right) \ltimes C^{\infty}\left(S^{1}\right) .
$$

An element of $\mathfrak{g}$ is a pair

$$
\left(f(x) \frac{d}{d x}, a(x)\right), \quad \text { where } \quad f(x) \frac{d}{d x} \in \operatorname{Vect}^{s}\left(S^{1}\right), \quad \text { and } \quad a(x) \in C^{\infty}\left(S^{1}\right) .
$$

It is known that this algebra has a three dimensional central extension given by the non-trivial cocycles

$$
\begin{aligned}
& \omega_{1}\left(\left(f(x) \frac{d}{d x}, a(x)\right),\left(g \frac{d}{d x}, b\right)\right)=\int_{S^{1}} f^{\prime}(x) g^{\prime \prime}(x) d x, \\
& \omega_{2}\left(\left(f(x) \frac{d}{d x}, a(x)\right),\left(g \frac{d}{d x}, b\right)\right)=\int_{S^{1}}\left[f^{\prime \prime}(x) b(x)-g^{\prime \prime}(x) a(x)\right] d x, \\
& \omega_{3}\left(\left(f(x) \frac{d}{d x}, a(x)\right),\left(g \frac{d}{d x}, b\right)\right)=2 \int_{S^{1}} a(x) b^{\prime}(x) d x .
\end{aligned}
$$

The first cocycle $\omega_{1}$ is the well-known Gelfand-Fuchs cocycle. The Virasoro algebra

$$
\operatorname{Vir}=\operatorname{Vect}^{s}\left(S^{1}\right) \oplus \mathbb{R}
$$

is the unique non-trivial central extension of $\operatorname{Vect}^{s}\left(S^{1}\right)$ based on the Gelfand-Fuchs cocycle. The space $C^{\infty}\left(S^{1}\right) \oplus \mathbb{R}$ is identified as regular part of the dual space to the Virasoro algebra. The pairing between this space and the Virasoro algebra is given by:

$$
\left\langle(u(x), a),\left(f(x) \frac{d}{d x}, a(x)\right)\right\rangle=\int_{S^{1}} u(x) f(x) d x+a \alpha .
$$

Similarly we consider the following extension of $\mathfrak{g}$,

$$
\widehat{\mathfrak{g}}=\operatorname{Vect}^{s}\left(S^{1}\right) \ltimes C^{\infty}\left(S^{1}\right) \oplus \mathbb{R}^{3} .
$$

The commutation relation in $\widehat{\mathfrak{g}}$ is given by

$$
\left[\left(f \frac{d}{d x}, a, \alpha\right),\left(g \frac{d}{d x}, b, \beta\right)\right]:=\left(\left(f g^{\prime}-f^{\prime} g\right) \frac{d}{d x}, f b^{\prime}-g a^{\prime}, \omega\right)
$$

where $\alpha=\left(\alpha_{1}, \alpha_{2}, \alpha_{3}\right), \beta=\left(\beta_{1}, \beta_{2}, \beta_{3}\right) \in \mathbb{R}^{3}$, and where $\omega=\left(\omega_{1}, \omega_{2}, \omega_{3}\right)$ are the cocycles.

Let

$$
\widehat{\mathfrak{g}}_{\mathrm{reg}}^{*}=C^{\infty}\left(S^{1}\right) \oplus C^{\infty}\left(S^{1}\right) \oplus \mathbb{R}^{3}
$$


denote the regular part of the dual space $\widehat{\mathfrak{g}}^{*}$ to the Lie algebra $\widehat{\mathfrak{g}}$, under the following pairing:

$$
\langle\widehat{u}, \widehat{f}\rangle=\int_{S^{1}}[f(x) u(x)+a(x) v(x)] d x+\alpha \cdot \gamma,
$$

where $\widehat{u}=(u(x), v, \gamma) \in \widehat{\mathfrak{g}}_{\text {reg }}^{*}, \widehat{f}=\left(f \frac{d}{d x}, a, \alpha\right) \in \widehat{\mathfrak{g}}$. Of particular interest are the coadjoint orbits in $\widehat{\mathfrak{g}}_{\text {reg. }}^{*}$. In this case, Gelfand, Vershik and Graev [9], have constructed some of the corresponding representations.

Let us introduce $H^{1}$ inner product on the algebra $\widehat{\mathfrak{g}}$

$$
\langle\widehat{f}, \widehat{g}\rangle_{H^{1}}=\int_{S^{1}}\left[f(x) g(x)+a(x) b(x)+\partial_{x} f(x) \partial_{x} g(x)\right] d x+\alpha \cdot \beta,
$$

where

$$
\widehat{f}=\left(f \frac{d}{d x}, a, \alpha\right), \quad \widehat{g}=\left(g \frac{d}{d x}, b, \beta\right) .
$$

Now we compute:

Lemma 1. The coadjoint operator with respect to the $H^{1}$ inner product is given by

$$
\operatorname{ad}_{\hat{f}}^{*}\left(\begin{array}{c}
u \\
v
\end{array}\right)=\left(\begin{array}{c}
\left(1-\partial^{2}\right)^{-1}\left[2 f^{\prime}(x)\left(1-\partial_{x}^{2}\right) u(x)+f(x)\left(1-\partial_{x}^{2}\right) u^{\prime}(x)+a^{\prime} v(x)\right] \\
f^{\prime} v(x)+f(x) v^{\prime}(x)
\end{array}\right) .
$$

Proof. Since we have identified $\mathfrak{g}$ with $\mathfrak{g}^{*}$, it follows from the definition that

$$
\begin{aligned}
\left\langle\operatorname{ad}_{\hat{f}}^{*} \widehat{u}, \widehat{g}\right\rangle_{H^{1}} & =\langle\widehat{u},[\widehat{f}, \widehat{g}]\rangle_{H^{1}} \\
& =-\int_{S^{1}}\left[\left(f g^{\prime}-f^{\prime} g\right) u-\left(f b^{\prime}-g a^{\prime}\right) v-\partial_{x}\left(f g^{\prime}-f^{\prime} g\right) \partial_{x} u\right] d x .
\end{aligned}
$$

After computing all the terms by integrating by parts and using the fact that the functions $f(x)$, $g(x), u(x)$ and $a(x), b(x), v(x)$ are periodic, the right hand side can be expressed as above.

Let us compute now the left hand side:

$$
\begin{aligned}
\operatorname{ad}_{\hat{f}}^{*}\left(\begin{array}{c}
u \\
v
\end{array}\right) & =\int_{S^{1}}\left[\left(\operatorname{ad}_{\hat{f}}^{*} u\right) g+\left(\operatorname{ad}_{\hat{f}}^{*} u\right)^{\prime} g^{\prime}+\left(\operatorname{ad}_{\hat{f}}^{*} v\right) b\right] d x \\
& =\int_{S^{1}}\left[\left[\left(1-\partial^{2}\right) \operatorname{ad}_{\hat{f}}^{*} u\right] g+\left(\operatorname{ad}_{\hat{f}}^{*} v\right) b\right] d x=\left\langle\left(\left(1-\partial^{2}\right) \operatorname{ad}_{\hat{f}}^{*} u,\left(\operatorname{ad}_{\hat{f}}^{*} v\right)\right),(g, b)\right\rangle .
\end{aligned}
$$

Thus by equating the the right and left hand sides, we obtain the desired formula.

We conclude that the Hamiltonian operator arising from the induced Lie-Poisson structure is

$$
\left(\begin{array}{cc}
D \rho+\rho D & v D \\
D v & 0
\end{array}\right)
$$

where $\rho=\left(1-\partial_{x}^{2}\right) u$. We conclude that

Theorem. A curve

$$
\widehat{c}(t)=\left(u(x, t) \frac{d}{d x}, v(x, t), \gamma\right) \subset \mathfrak{g}
$$

defines a geodesic in the $H^{1}$ metric if and only if

$$
\begin{aligned}
& u_{t}-u_{x x t}=3 u u_{x}+v v_{x}-\left(u u_{x x}+\frac{1}{2} u_{x}^{2}\right)_{x}, \\
& v_{t}=2(u v)_{x} .
\end{aligned}
$$




\section{Geodesic flow and superintegrable systems}

The first and foremost characteristic property of a superalgebra is that all the additive groups of its basic and derived structures are $\mathbb{Z}_{2}$ graded. A vector superspace is a $\mathbb{Z}_{2}$ graded vector space $V=V_{B} \oplus V_{F}$. An element $v$ of $V_{B}$ (resp. $V_{F}$ ) is said to be even or bosonic (resp. odd or fermionic). The super-commutator of a pair of elements $v, w \in V$ is defined to be the element

$$
[v, w]=v w-(-1)^{\bar{v} \bar{w}} w v .
$$

The generalized Neveu-Schwartz superalgebra [24] is composed of two parts: the bosonic (even) and the fermionic (odd). These are given by

$$
S \mathfrak{g}_{B}=\operatorname{Vect}^{s}\left(S^{1}\right) \oplus C^{\infty}\left(S^{1}\right) \oplus \mathbb{R}^{3}, \quad S \mathfrak{g}_{F}=C^{\infty}\left(S^{1}\right) \oplus C^{\infty}\left(S^{1}\right) .
$$

There are three different actions:

(A) the action of the bosonic part on the bosonic part, discussed earlier.

(B) the action of the bosonic part on the fermionic part, given by

$$
\begin{aligned}
& {[,]: S \mathfrak{g}_{B} \otimes S \mathfrak{g}_{F} \longrightarrow S \mathfrak{g}_{F},} \\
& {\left[\left(f(x) \frac{d}{d x}, a(x)\right),(\phi(x), \alpha(x))\right]:=\left(\begin{array}{c}
f(x) \phi^{\prime}-\frac{1}{2} f^{\prime}(x) \phi(x) \\
f(x) \alpha^{\prime}(x)+\frac{1}{2} f^{\prime}(x) \alpha(x)-\frac{1}{2} a^{\prime}(x) \phi(x)
\end{array}\right) .}
\end{aligned}
$$

(C) the action of the fermionic part on the fermionic part, given by

$$
\begin{aligned}
& {[,]_{+}: S \mathfrak{g}_{F} \otimes S \mathfrak{g}_{F} \longrightarrow S \mathfrak{g}_{B},} \\
& {[(\phi(x), \alpha(x)),(\psi(x), \beta(x))]_{+}=\left(\phi \psi \frac{d}{d x}, \phi \beta+\alpha \psi, \omega_{F}\right),}
\end{aligned}
$$

where $\omega_{F}=\left(\omega_{F 1}, \omega_{F 2}, \omega_{F 3}\right)$ is the fermionic cocycle, with components

$$
\begin{aligned}
& \omega_{F 1}((\phi, \alpha),(\psi, \beta))=2 \int_{S^{1}} \phi^{\prime}(x) \psi^{\prime}(x) d x, \\
& \omega_{F 2}((\phi, \alpha),(\psi, \beta))=-2 \int_{S^{1}}\left(\phi^{\prime}(x) \beta(x)+\psi^{\prime} \alpha(x)\right) d x, \\
& \omega_{F 3}((\phi, \alpha),(\psi, \beta))=4 \int_{S^{1}} \alpha(x) \beta(x) d x .
\end{aligned}
$$

The supercocycle $\omega_{S}$ has two parts, the bosonic and the fermionic:

$$
\omega_{S}=\omega_{B} \oplus \omega_{F},
$$

where the bosonic part $\omega_{B}$ is identical to $\omega=\left(\omega_{1}, \omega_{2}, \omega_{3}\right)$, as given by $(3)$.

With this in hand, we establish the supersymmetric 2-component generalization of the Camassa-Holm equation.

Definition. The $H^{1}$ pairing between the regular part of the dual space $S \widehat{\mathfrak{g}}^{*}$ and $S \mathfrak{g}$ is given by

$$
\begin{aligned}
& \left\langle(u(x), v(x), \psi(x), \beta),\left(f(x) \frac{d}{d x}, a(x), \phi(x), \alpha\right)\right\rangle_{H^{1}} \\
& =\int_{S^{1}} f(x) u(x) d x+\int_{S^{1}} f_{x} u_{x} d x+\int_{S^{1}} a(x) v(x) d x \\
& \quad+\int_{S^{1}} \phi(x) \psi(x) d x+\int_{S^{1}} \phi_{x} \psi_{x} d x+\int_{S^{1}} \alpha(x) \beta(x) d x .
\end{aligned}
$$


Let us compute the coadjoint action with respect to the $H^{1}$ norm.

\section{Lemma 2.}

$$
\begin{aligned}
& \operatorname{ad}_{\hat{f}}^{*}\left(\begin{array}{c}
u(x) \\
v(x) \\
\psi(x) \\
\beta(x)
\end{array}\right) \\
& =\left(\begin{array}{c}
\left(1-\partial^{2}\right)^{-1}\left[2 f^{\prime}\left(1-\partial^{2}\right) u(x)+\left(1-\partial^{2}\right) u^{\prime} f+a^{\prime} v+\frac{1}{2}\left(1-\partial^{2}\right) \psi^{\prime} \phi+\frac{3}{2}\left(1-\partial^{2}\right) \psi \phi^{\prime}\right] \\
f^{\prime} v+f v^{\prime}+\frac{1}{2}\left(\beta^{\prime} \phi+\beta \phi^{\prime}\right) \\
\left(1-\partial^{2}\right)^{-1}\left[f\left(1-\partial^{2}\right) \psi^{\prime}+\frac{3}{2} f^{\prime}\left(1-\partial^{2}\right) \psi+\frac{1}{2} a^{\prime} \beta+\left(1-\partial^{2}\right) u \phi+v \alpha\right] \\
f \beta^{\prime}+\frac{1}{2} f^{\prime} \beta+v \phi
\end{array}\right) .
\end{aligned}
$$

Sketch of the proof. Using the definition of the coadjoint action

$$
\left\langle\operatorname{ad}_{\hat{f}}^{*} \widehat{u}, \widehat{g}\right\rangle_{H^{1}}=\langle\widehat{f},[\widehat{u}, \widehat{g}]\rangle_{H^{1}}
$$

with

$$
\widehat{f}=\left(\begin{array}{c}
f(x) \\
a(x) \\
\phi(x) \\
\alpha(x)
\end{array}\right), \quad \widehat{u}=\left(\begin{array}{c}
u(x) \\
v(x) \\
\psi(x) \\
\beta(x)
\end{array}\right), \quad \widehat{g}=\left(\begin{array}{c}
g(x) \\
b(x) \\
\chi(x) \\
\gamma(x)
\end{array}\right),
$$

we obtain

$$
\left.\langle(u, v, \psi, \beta)],\left(\begin{array}{c}
\left(f g^{\prime}-f^{\prime} g\right) \frac{d}{d x}+\phi \chi \frac{d}{d x} \\
f b^{\prime}-g a^{\prime}+\phi \gamma+\alpha \chi \\
f \chi^{\prime}-\frac{1}{2} f^{\prime} \chi+g \phi^{\prime}-\frac{1}{2} g^{\prime} \phi \\
f \gamma^{\prime}+\frac{1}{2} f^{\prime} \gamma-\frac{1}{2} a^{\prime} \gamma+g \alpha^{\prime}+\frac{1}{2} g^{\prime} \alpha-\frac{1}{2} b^{\prime} \phi
\end{array}\right)\right\rangle
$$

This would give us the right hand side without the $\left(1-\partial^{2}\right)^{-1}$ term, which appears on the left hand side:

$$
\begin{aligned}
\text { L.H.S. }= & \int_{S^{1}}\left(\operatorname{ad}_{\hat{f}}^{*} u\right) g d x+\int_{S^{1}}\left(\operatorname{ad}_{\hat{f}}^{*} u\right)^{\prime} g^{\prime} d x \int_{S^{1}}\left(\operatorname{ad}_{\hat{f}}^{*} v\right) b d x \\
& +\int_{S^{1}}\left(\operatorname{ad}_{\hat{f}}^{*} \psi\right) \phi d x+\int_{S^{1}}\left(\operatorname{ad}_{\hat{f}}^{*} \psi^{\prime}\right) \phi^{\prime} d x+\int_{S^{1}}\left(\operatorname{ad}_{\hat{f}}^{*} \beta\right) \alpha d x \\
= & \int_{S^{1}}\left[\left(1-\partial^{2}\right) \operatorname{ad}_{\hat{f}}^{*} u\right] g d x+\int_{S^{1}}\left(\operatorname{ad}_{\hat{f}}^{*} v\right) b d x \\
& +\int_{S^{1}}\left[\left(1-\partial^{2}\right) \operatorname{ad}_{\hat{f}}^{*} \psi\right] \phi d x+\int_{S^{1}}\left(\operatorname{ad}_{\hat{f}}^{*} \beta\right) \alpha d x .
\end{aligned}
$$

Equating the right and left hand sides, we obtain the desired formula.

Therefore, if we use the Euler-Poincaré equation and the computational trick used in [5], we obtain the supersymmetric version of the two component Camassa-Holm equation:

$$
\begin{aligned}
& m_{t}=2 m u_{x}+m_{x} u+\left(v v_{x}\right)+3 \xi \xi^{\prime \prime} \\
& v_{t}=2(u v)_{x}+\beta^{\prime} \xi^{\prime}+\beta \xi^{\prime \prime} \\
& \left(1-\partial^{2}\right) \xi_{t}=4 m \xi^{\prime}+3 m^{\prime} \xi+2 \xi^{\prime \prime \prime}, \\
& \beta_{t}=2 u \beta^{\prime}+u^{\prime} \beta+2 v \xi^{\prime}
\end{aligned}
$$

where $m=u-u_{x x}$. 


\section{Corollary 1.}

$$
\operatorname{ad}_{\hat{f}}^{*} \widehat{u}=\left(\begin{array}{c}
2 u f^{\prime}(x)+u^{\prime} f+a^{\prime} v+f^{\prime \prime \prime}+\frac{1}{2} \psi^{\prime} \phi+\frac{3}{2} \psi \phi^{\prime} \\
f^{\prime} v+f v^{\prime}+\frac{1}{2}\left(\beta^{\prime} \phi+\beta \phi^{\prime}\right) \\
f \psi^{\prime}+\frac{3}{2} f^{\prime} \psi+\frac{1}{2} a^{\prime} \beta+u \phi+v \alpha+2 \phi^{\prime \prime} \\
f \beta^{\prime}+\frac{1}{2} f^{\prime} \beta+v \phi
\end{array}\right) .
$$

In this way, we recover a supersymmetric version of Ito equation [14] given by

$$
\begin{aligned}
& u_{t}=6 u u_{x}+2\left(v v_{x}\right)+u_{x x x}+3 \xi \xi^{\prime \prime}, \\
& v_{t}=2(u v)_{x}+\beta^{\prime} \xi^{\prime}+\beta \xi^{\prime \prime} \\
& \xi_{t}=4 u \xi^{\prime}+3 u^{\prime} \xi+2 \xi^{\prime \prime \prime} \\
& \beta_{t}=2 u \beta^{\prime}+u^{\prime} \beta+2 v \xi^{\prime} .
\end{aligned}
$$

Corollary 2. In the supersymmetric Ito equation (4):

(A) if we set the super variables $\xi=\beta=0$, we recover the Ito equation.

(B) If we set $v=\beta=0$, we obtain

$$
\begin{aligned}
& u_{t}=6 u u_{x}+u_{x x x}+3 \xi \xi^{\prime \prime}, \\
& \xi_{t}=4 u \xi^{\prime}+3 u^{\prime} \xi+2 \xi^{\prime \prime \prime},
\end{aligned}
$$

which is a fermionic extension of $K d V$ equation and, modulo rescalings, is the super KdV equation of Mathieu and Manin-Radul [17, 19].

Remark 2. The physicists usually distinguish the fermionic and supersymmetric extension among each other. From the physical point of view the supersymmetry requires the invariance under the supersymmetric transformations while for the fermionic extension we have no such restriction. All extended equations considered in this paper are fermionic.

\section{Acknowledgements}

One of the authors (PG) would like to thank Professor Andy Hone for various information about the two component Camassa-Holm equation. PG is also grateful to Professor Valentin Ovsienko for various stimulating discussions. PG would like to thank Professor Dieter Mayer at TU Clausthal, where the part of work was done in a stimulating atmosphere. This work was partially supported by the DFG Research Group "Zeta functions and locally symmetric spaces" which is gratefully acknowledged.

The work of the second author was supported in part by NSF Grant DMS 05-05293.

[1] Arbarello E., De Concini C., Kac V.G., Procesi C., Moduli space of curves and representation theory, Comm. Math. Phys., 1988, V.117, 1-36.

[2] Camassa R., Holm D.D., An integrable shallow water equation with peaked solitons, Phys. Rev. Lett., 1993, V.71, 1661-1664, patt-sol/9305002.

[3] Camassa R., Holm D.D., Hyman J.M., A new integrable shallow water equation, Adv. in Appl. Mech., 1994, V.31, 1-33.

[4] Chen M., Liu S.-Q., Zhang Y., A 2-component generalization of the Camassa-Holm equation and its solutions, nlin.SI/0501028.

[5] Devchand C., Schiff J., The supersymmetric Camassa-Holm equation and geodesic flow on the superconformal group, J. Math. Phys., 2001, V.42, 260-273, solv-int/9811016.

[6] Ebin D., Marsden J., Groups of diffeomorphisms and the motion of an incompressible fluid, Ann. Math., 1970, V.92, 102-163.

[7] Falqui G., On a Camassa-Holm type equation with two dependent variables, nlin.SI/0505059. 
[8] Fuchssteiner B., Some tricks from the symmetry-toolbox for nonlinear equations: generalizations of the Camassa-Holm equation, Phys. D, 1996, V.95, 229-243.

[9] Gelfand I.M., Graev I.M., Vershik A.M., Models of representations of current groups, in Representations of Lie Groups and Lie Algebras, Budapest, Akad. Kiad, 1985, 121-179.

[10] Guha P., Integrable geodesic flows on the (super)extension of the Bott-Virasoro group, Lett. Math. Phys., 2000, V.52, 311-328.

[11] Guha P., Geodesic flows, bi-Hamiltonian structure and coupled KdV type systems, J. Math. Anal. Appl., 2005, V.310, 45-56.

[12] Guha P., Euler-Poincaré formalism of coupled KdV type systems and diffeomorphism group on $S^{1}, J$. Appl. Anal., 2005, V.11, 261-282.

[13] Holm D.D., Marsden J.E., Ratiu T.S., The Euler-Poincaré equations and semidirect products with applications to continuum theories, Adv. Math., 1998, V.137, 1-81, chao-dyn/9801015.

[14] Ito M., Symmetries and conservation laws of a coupled nonlinear wave equation, Phys. Lett. A, 1982, V.91, $335-338$.

[15] Li Y.A., Olver P.J., Rosenau P., Non-analytic solutions of nonlinear wave models, in Nonlinear Theory of Generalized Functions, Editors M. Grosser, G. Hörmann, M. Kunzinger and M. Oberguggenberger, Research Notes in Mathematics, Vol. 401, New York, Chapman and Hall/CRC, 1999, 129-145.

[16] Liu S.-Q., Zhang Y., Deformations of semisimple bi-Hamiltonian structures of hydrodynamic type, J. Geom. Phys., 2005, V.54, 427-453, math.DG/0405146.

[17] Manin Yu.I., Radul A.O., A supersymmetric extension of the Kadomtsev-Petviashvili hierarchy, Comm. Math. Phys., 1985, V.98, 65-77.

[18] Marcel P., Ovsienko V., Roger C., Extension of the Virasoro and Neveu-Schwartz algebras and generalized Sturm-Liouville operators, Lett. Math. Phys., 1997, V.40, 31-39, hep-th/9602170.

[19] Mathieu P., Supersymmetric extension of the Korteweg-de Vries equation, J. Math. Phys., 1988, V.29, 2499-2506.

[20] Misiolek G., A shallow water equation as a geodesic flow on the Bott-Virasoro group, J. Geom. Phys., 1998, V.24, 203-208.

[21] Olver P.J., Applications of Lie groups to differential equations, 2nd ed., Graduate Texts in Mathematics, Vol. 107, New York, Springer-Verlag, 1993.

[22] Olver P.J., Rosenau P., Tri-Hamiltonian duality between solitons and solitary-wave solutions having compact support, Phys. Rev. E, 1996, V.53, 1900-1906.

[23] Ovsienko V.Yu., Khesin B.A., KdV super equation as an Euler equation, Funct. Anal. Appl., 1987, V.21, $329-331$.

[24] Ovsienko V.Yu., Coadjoint representation of Virasoro-type Lie algebras and differential operators on tensordensities, in Infinite Dimensional Kähler Manifolds (1995, Oberwolfach), DMV Sem., Vol. 31, Basel, Birkhäuser, 2001, 231-255.

[25] Popowicz Z., A 2-component or $N=2$ supersymmetric Camassa-Holm equation, nlin.SI/0509050.

[26] Rosenau P., Nonlinear dispersion and compact structures, Phys. Rev. Lett., 1994, V.73, $1737-1741$. 